\title{
Thromboprophylaxis in Intramedullary Limb Lengthening Surgery
}

\author{
Alexios D lliadis ${ }^{1}$, Anna Timms², Sharron Fugazzotto ${ }^{3}$, Penina Edel ${ }^{4}$, Simon Britten ${ }^{5}$, Jonathan Wright ${ }^{6}$, David Goodier ${ }^{7}$, \\ Peter Calder ${ }^{8}$
}

\begin{abstract}
Aim: The use of intramedullary lengthening devices is becoming increasingly popular. There are limited data regarding the incidence of venous thromboembolism following intramedullary lengthening surgery and no reports or guidance for current practice on use of thromboprophylaxis. Following a case of post-operative deep vein thrombosis in our institution, we felt that it is important to assess best practice. We conducted a national survey to collect data that would describe current practice and help develop consensus for treatment.

Materials and methods: We identified surgeons across the UK that perform adult intramedullary limb lengthening through the British Limb Reconstruction Society membership and a Precise Users database. Surgeons were contacted and asked to respond to an online survey. Responses to thromboprophylaxis regimes employed in their practice and cases of venous thromboembolism were collated.

Results: 24 out of 54 surgeons identified responded with a total of 454 cases of adult intramedullary lengthening ( 352 femoral and 102 tibial nails) performed over a five year period (January 2015-January 2020). Only one case of deep venous thrombosis (DVT) following femoral lengthening was reported. There is wide variability in practice both in terms of thromboprophylaxis risk assessment, choice of medications and duration of treatment. The vast majority of surgeons (85\%) felt that there was insufficient evidence available to guide their practice.

Conclusions: Intramedullary lengthening is a surgical treatment growing in popularity. There are limited data available to guide decision-making regarding aspects of treatment such as thromboprophylaxis. This is reflected in the wide variation in practice reported in this study. There are both a need and a desire to gather data that will allow us to come to a consensus and to guide safe practice.

Clinical significance:Venous thromboembolism is a potential complication of lower limb lengthening surgery. We report on national incidence and current practices of thromboprophylaxis to allow for informed decision-making and help develop consensus for best practice.

Keywords: Complication, Cosmetic limb lengthening, Internal lengthening nail, Intramedullary lengthening, Limb lengthening, PRECICE.

Strategies in Trauma and Limb Reconstruction (2020): 10.5005/jp-journals-10080-1505
\end{abstract}

\section{INTRODUCTION}

Following introduction of the concept of distraction osteogenesis by llizarov, ${ }^{1,2}$ advances in both technique and technology have resulted in the development of fully implantable intramedullary lengthening nails. ${ }^{3}$ The most popular device currently is the PRECICE Intramedullary Limb-Lengthening System (ILLS) (Ellipse Technologies Inc. CA, US). ${ }^{4}$

Venous thromboembolism (VTE) is a potential complication of any lower limb orthopaedic surgery with variable rates reported. 5,6 There are national guidelines with regards to the use of thromboprophylaxis in orthopaedic surgery provided by the National Institute for Health and Clinical Excellence (NICE) with specific recommendations for certain commonly performed orthopaedic procedures such as lower limb arthroplasty and neck of femur fractures. ${ }^{7}$ A VTE risk assessment tool has been developed to allow clinicians to determine the need for thromboprophylaxis in other types of surgery (Table 1$){ }^{8}$

Previous studies have reported low rates of VTE in patients undergoing limb lengthening and deformity correction using external fixator frames and have recommended on the safety of employing the NICE risk assessment tool for decision making. ${ }^{9}$ There is limited information available with regards to its incidence following surgery for insertion of femoral and tibial intramedullary lengthening nails as most studies report on small cohorts of patients. $^{10-13}$
1-4,6-8 Limb Reconstruction Unit, Royal National Orthopaedic Hospital
NHS Trust, Stanmore, United Kingdom
${ }^{5}$ Limb Reconstruction Unit, Leeds Teaching Hospitals NHS Trust, Leeds,
United Kingdom

Corresponding Author: Alexios D Iliadis, Paediatric Unit, Royal National Orthopaedic Hospital NHS Trust, Stanmore, United Kingdom, Phone: +44 2039470100, e-mail: alexis-dimitris.iliadis@nhs.net

How to cite this article: Iliadis $A D$, Timms A, Fugazzotto $S$, et al. Thromboprophylaxis in Intramedullary Limb Lengthening Surgery. Strategies Trauma Limb Reconstr 2020;15(3):151-156.

Source of support: Nil

Conflict of interest: None

Our unit is one of the largest centers utilising the PRECICE ILLS, with over 150 cases performed in adults over the past 5 years. In our practice thromboprophylaxis is prescribed following risk assessment and routinely consists of the use of peri-operative intermittent pneumatic compression on the contralateral side, use of thrombo-embolism deterrent (TED) stockings for a 2 week period following surgery and the use of weight-adjusted low molecular weight heparin (LMWH) for a variable period until the patient is deemed safe for discharge by physiotherapy (safe in transfer, independent in mobility with support, adequate range 
Table1: VTE risk assessment tool from Department of Health, National Institute of Clinical Excellence ${ }^{8}$

\begin{tabular}{lll}
\hline Mobility - all patients (tick one box) & Tick & Tick \\
\hline Surgical patient & Medical patient expected to & Tick \\
& have ongoing reduced mobility \\
relative to normal state & $\begin{array}{l}\text { significantly reduced mobility relative to } \\
\text { normal state }\end{array}$
\end{tabular}

Assess for thrombosis and bleeding risk below Risk assessment now complete

\begin{tabular}{|c|c|c|c|}
\hline \multicolumn{4}{|l|}{ Thrombosis risk } \\
\hline Patient related & Tick & Admission related & Tick \\
\hline Active cancer or cancer treatment & & $\begin{array}{l}\text { Significantly reduced mobility for } 3 \text { days or } \\
\text { more }\end{array}$ & \\
\hline Age $>60$ & & Hip or knee replacement & \\
\hline Dehydration & & Hip fracture & \\
\hline Known thrombophilias & & Total anaesthetic + surgical time $>90 \mathrm{~min}$ & \\
\hline Obesity (BMI > 30 kg/m²) & & $\begin{array}{l}\text { Surgery involving pelvis or lower limb with a } \\
\text { total anaesthetic + surgical time }>60 \mathrm{~min}\end{array}$ & \\
\hline $\begin{array}{l}\text { One or more significant medical comorbidities } \\
\text { (e.g. heart disease; metabolic, endocrine or } \\
\text { respiratory pathologies; acute infectious diseases; } \\
\text { inflammatory conditions) }\end{array}$ & & $\begin{array}{l}\text { Acute surgical admission with inflammatory or } \\
\text { intra-abdominal condition }\end{array}$ & \\
\hline $\begin{array}{l}\text { Personal history or first-degree relative with a } \\
\text { history of VTE }\end{array}$ & & Critical care admission & \\
\hline Use of hormone replacement therapy & & Surgery with significant reduction in mobility & \\
\hline \multicolumn{4}{|l|}{$\begin{array}{l}\text { Use of oestrogen-containing contraceptive } \\
\text { therapy }\end{array}$} \\
\hline \multicolumn{4}{|l|}{ Varicose veins with phlebitis } \\
\hline \multicolumn{4}{|l|}{$\begin{array}{l}\text { Pregnancy or }<6 \text { weeks post-partum (see NICE } \\
\text { guidance for specific risk factors) }\end{array}$} \\
\hline \multicolumn{4}{|l|}{ Bleeding risk } \\
\hline Patient related & Tick & Admission related & Tick \\
\hline Active bleeding & & Neurosurgery, spinal surgery or eye surgery & \\
\hline $\begin{array}{l}\text { Acquired bleeding disorders (such as acute liver } \\
\text { failure) }\end{array}$ & & Other procedure with high bleeding risk & \\
\hline $\begin{array}{l}\text { Concurrent use of anticoagulants known to } \\
\text { increase the risk of bleeding (such as warfarin with } \\
\text { INR }>2 \text { ) }\end{array}$ & & $\begin{array}{l}\text { Lumbar puncture/epidural/spinal anaesthesia } \\
\text { expected within the next } 12 \mathrm{hr}\end{array}$ & \\
\hline Acute stroke & & $\begin{array}{l}\text { Lumbar puncture/epidural/spinal anaesthesia } \\
\text { within the previous } 4 \mathrm{hr}\end{array}$ & \\
\hline \multicolumn{4}{|l|}{ Thrombocytopaenia (platelets $<75 \times 10^{9} / \mathrm{L}$ ) } \\
\hline \multicolumn{4}{|l|}{$\begin{array}{l}\text { Uncontrolled systolic hypertension } \\
\text { (230/120 mmHg or higher) }\end{array}$} \\
\hline $\begin{array}{l}\text { Untreated inherited bleeding disorders (such as } \\
\text { haemophilia and von Willebrand's disease) }\end{array}$ & & & \\
\hline
\end{tabular}

of movement). Employing this regime we have encountered one case of DVT following surgery for femoral intramedullary nail insertion.

The aim of this study was to report the national incidence of DVT in adult patients undergoing PRECICE ILLS. To report on surgeon preference in DVT prevention and determine if there is sufficient guidance to allow confidence in thromboprophylaxis decision making.

\section{Materials and Methods}

Following the occurrence of VTE in one of our patients, a Serious Incident Investigation Report was conducted. We performed a retrospective review of all adult cases who underwent surgery with the PRECICE ILLS in our institution by searching through patient electronic records to determine the incidence of VTE and the thromboprophylaxis regime employed.

We subsequently conducted a national survey. United Kingdom (UK) based surgeons who perform intramedullary lengthening surgery were identified through the registry of the British Limb Reconstruction Society (BLRS) and the PRECICE users database. They were asked to complete an anonymised online survey through SurveyMonkey (SVMK Inc. CA, US). The survey consisted of open ended, multiple choice and Likert scale questions regarding the use of thromboprophylaxis following femoral and tibial intramedullary lengthening surgery and the incidence of 
Table 2: Survey questionnaire

Q1: In the last 5 years, what is the total number of cases of FEMORAL intramedullary lengthening surgery that you have performed in adult patients?

Q2: In the last 5 years, what is the total number of cases of TIBIAL intramedullary lengthening surgery that you have performed in adult patients?

Q3: In your practice, how many cases of DVT or PE have you observed following FEMORAL intramedullary lengthening surgery in the last 5 years?

Q4: In your practice, how many cases of DVT or PE have you observed following TIBIAL intramedullary lengthening surgery in the last 5 years?

Q5: In cases of DVT or PE, did the patients receive any form of thromboprophylaxis postoperative?

Q6: Do you routinely use thromboprophylaxis following FEMORAL intramedullary lengthening surgery in adults?

Q7: If using TEDS, for how many days post-surgery?

Q8: If using chemical thromboprophylaxis, what is your medication of choice and how many days of prescription?

Q9: Do you routinely use thromboprophylaxis following TIBIAL

intramedullary lengthening surgery in adults?

Q10: If using TEDS, for how many days post-surgery?

Q11: If using chemical thromboprophylaxis, what is your medication of choice and how many days of prescription?

Q12: If using chemical thromboprophylaxis, do you give everyone the same dose or do you adjust for patients, weight?

Q13: I feel that there is strong evidence/level of recommendation to support my practice regarding the use of thromboprophylaxis following intramedullary lengthening surgery.

venous thromboembolism in their adult practice over a five year period (January 2015-February 2020). Table 2 shows the survey questions.

\section{Results}

On the 2nd of July 2019, a 41 year old male was admitted to our institution for elective right femoral de-rotation osteotomy and insertion of PRECICE ILLS. He had no relevant comorbidities and was identified as low risk for VTE as per NICE risk assessment tool (Table 2). A decision to prescribe LMWH (Tinzaparin Sodium) post-operatively was made as his surgery was prolonged to over 90 minutes due to the intramedullary nail failing to lengthen when initially tested. The nail was removed and replaced with a new implant. Post-operatively, mobility was delayed secondary to numbness in the patient's limbs following an epidural infusion for pain relief. The patient then felt unwell and displayed anxiety over the equipment used to aid mobilisation. The psychology liaison team became involved due to this mood change. In our unit, the average length of stay following this surgery is four days, but in this case the total length of stay was extended to 20 days. Throughout the stay the patient received Tinzaparin daily as well as mechanical prophylaxis in the form of TEDS. His weight was $>100 \mathrm{~kg}$ on admission and therefore a twice daily dose of 4500 units is recommended. ${ }^{14}$ During the first three days following surgery the patient only received a single daily dose. The prescription was then changed to the recommended dose. He was discharged home independently mobile at which point his prescription was stopped. One week following discharge (31/07/2019) he presented to his local hospital with symptoms of calf pain and dyspnoea. He was diagnosed with a right below knee DVT and bilateral Pulmonary Emboli (PE) for which he was commenced on treatment. There were no further complications in his care, he completed his anticoagulation course and fully recovered. The lengthening process was otherwise uneventful with full consolidation of the regenerate and no soft tissue contractures.

A retrospective audit in our institution, identified 162 adult patients that have undergone PRECICE ILLS during the five year period (January 2015-January 2020). Following review of their electronic records, we did not identify any further cases of VTE.

Fifty-four surgeons across the UK were identified, who have performed surgery with intramedullary lengthening devices during this five year period (Jan 2015-Feb 2020). Twenty four of those identified responded with a total of 454 cases of intramedullary lengthening surgery (352 femoral nails and 102 tibial nails) performed in adults over the last 5 years. According to the PRECICE users' database, a total of 623 intramedullary lengthening devices have been implanted in the UK for this period including both adults and children.

\section{Thromboprophylaxis Following Femoral PRECICE ILLS}

$80 \%$ of respondents routinely employ both chemical and mechanical prophylaxis whilst $20 \%$ would only prescribe chemical prophylaxis in the presence of risk factor identification during screening. With regards to the length of mechanical prophylaxis (TEDS) prescription, some clinicians only prescribe for 48 hours post-operatively whilst others prescribe for up to 6 weeks (Fig. 1). With regards to the choice and duration of chemical prophylaxis there is again wide variation in practice (Fig. 2).

\section{Thromboprophylaxis Following Tibia PRECICE ILLS}

$80 \%$ of respondents again routinely employ both chemical and mechanical prophylaxis, with the remaining $20 \%$ only prescribing chemical prophylaxis in the presence of risk factor identification during screening. Further variations in practice with regards to length of treatment and choice of prophylaxis also exist as demonstrated (Figs 3 and 4).

Further variability in prescribing practice of chemical thromboprophylaxis is highlighted with regards to dose adjustment

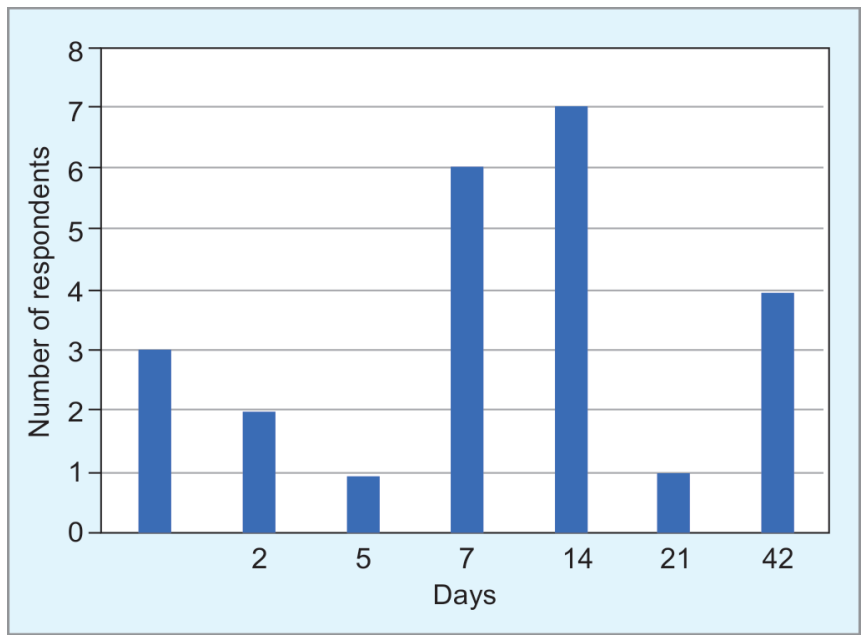

Fig. 1: Mechanical prophylaxis (TEDS) duration following femoral intramedullary lengthening nail insertion 


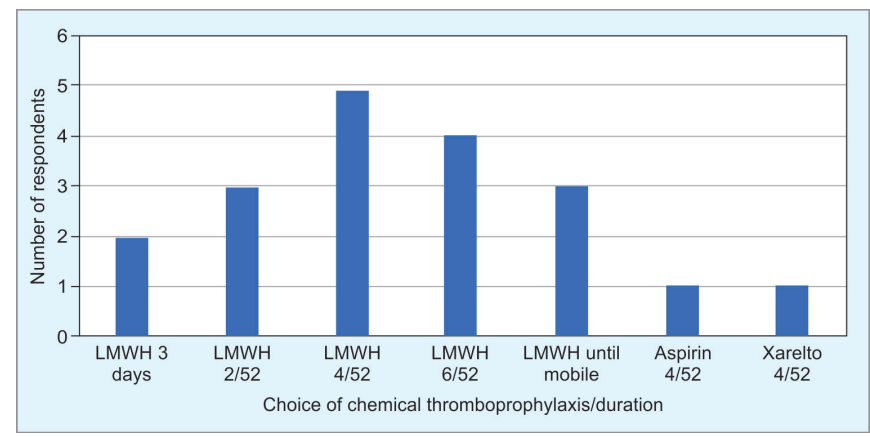

Fig. 2: Chemical thromboprophylaxis choice and duration following femoral intramedullary lengthening nail insertion

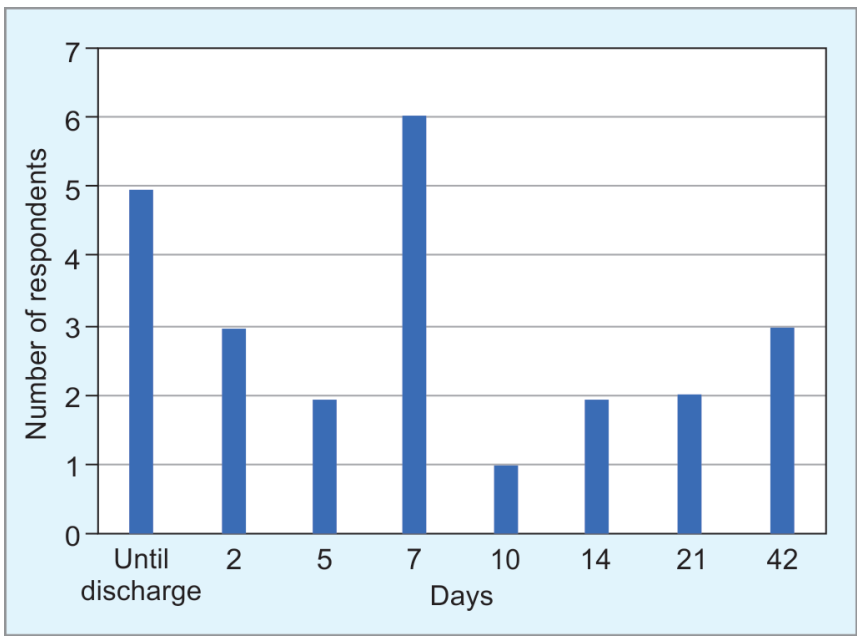

Fig. 3: Mechanical prophylaxis (TEDS) duration following tibial intramedullary lengthening nail insertion

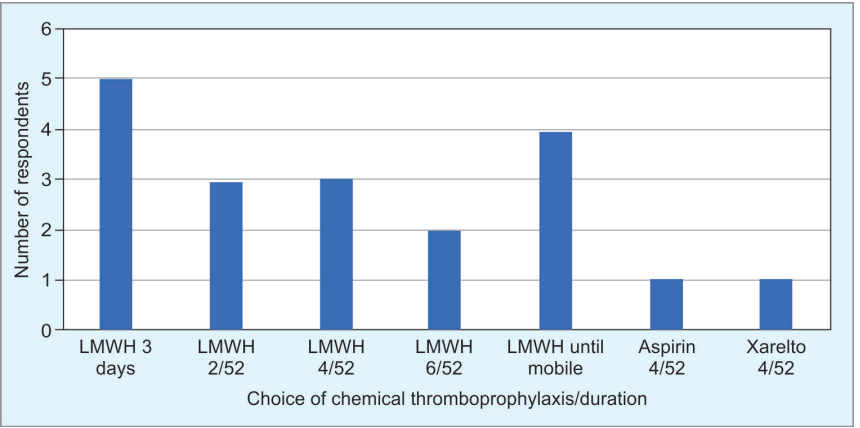

Fig. 4: Chemical thromboprophylaxis choice and duration following tibial intramedullary lengthening nail insertion

as per weight, with $45 \%$ of respondents adjusting dose as per patients' weight and $55 \%$ prescribing a standard dose for all patients.

When asked about level of evidence available, only $20 \%$ of respondents felt that there is sufficient evidence to guide them on their decision making process of prescribing thromboprophylaxis (Fig. 5).

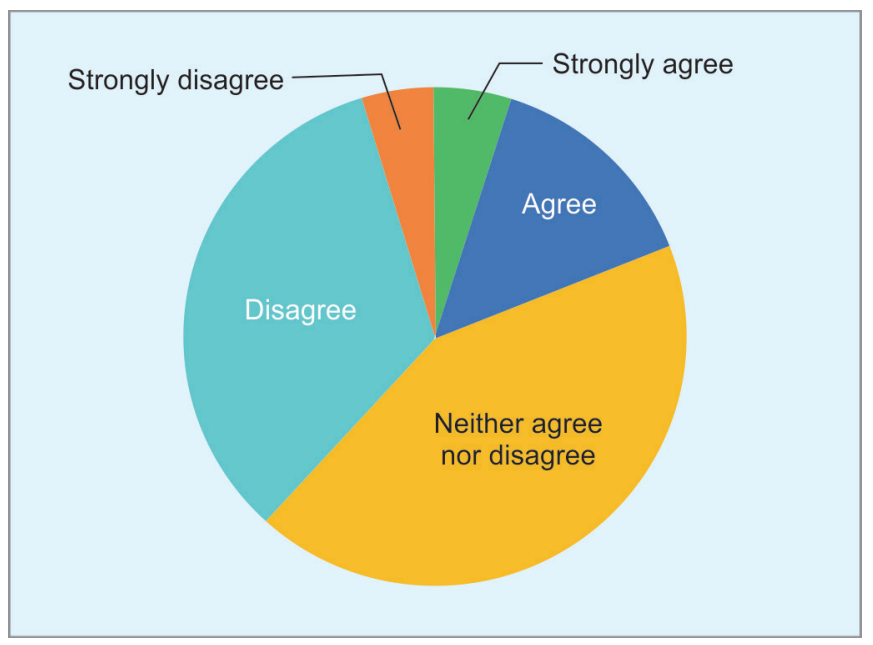

Fig. 5: Responses to question 13 Likert scale :"I feel that there is strong evidence / level of recommendation to support my practice regarding the use of thromboprophylaxis following intramedullary lengthening surgery"

\section{Discussion}

Responses to our survey show a low incidence of DVT in adult patients undergoing limb lengthening using the PRECICE ILLS. Our results are in keeping with low incidence reported in other series. $^{10-13}$ In the absence of the pathophysiologic processes included in Virchow's triad (vascular endothelial damage, stasis of blood flow and hypercoagulability of blood), thrombus does not usually develop. ${ }^{15}$ There are various differences in this type of surgery that may predispose patients to lower risk as compared to other orthopaedic lower limb operations. There is minimal period of immobilisation and bed rest and therefore venous stasis. There is minimal limb manipulation and potentially less endothelial vascular injury. There is no significant trauma as controlled minimally invasive corticotomies are performed and therefore, there is less systemic hypercoagulability response. The recent development of devices (STRYDE PRECICE ILLS, Nuvasive Inc., CA, US), that may allow immediate full weight bearing and encouragement of the "normal" physiological use of the limb, may further reduce the risk of DVT.

There are several limitations in this study. It was conducted as a retrospective report on surgeons experience through an anonymised online survey. There is an inherent risk of recall bias. Furthermore, there was a low response rate with approximately $50 \%$ of invited clinicians responding. Despite this, the number of procedures reported consist of over $70 \%$ of the 623 nails implanted in the UK during this period as per the manufacturer. Considering that this number includes both adult and paediatric patients, our study is likely reporting on an even higher percentage of cases performed. There is a degree of selection bias inherent on this type of surgery with the majority of patients undergoing it in our institution, being relatively "fit" without major co-morbidities and with independence in mobility.

Responses indicate that there is wide variability in clinical practice with regards to the choice of thromboprophylaxis in these patients both with regards to the regime employed as well as with regards to duration of treatment. Furthermore a substantial proportion of responding clinicians feel that this 
variation in practice is due to lack of consensus and available evidence.

Similar uncertainty maybe seen in other orthopaedic patient groups. There is lack of consensus with regards to the clinical benefit of routine thromboprophylaxis in the management of isolated tibia fractures with a recent meta-analysis suggesting the absence of treatment effect of routine anticoagulation following the surgical management of these injuries. ${ }^{16}$ High rates of VTE have been reported with femoral fractures ${ }^{17,18}$ but there is still debate with regards to the optimal use of routine prophylaxis regarding both choice and duration of treatment.

Even for those groups of orthopaedic patients where a consensus for thromboprophylaxis exists (lower limb arthroplasty and neck of femur fractures), there are aspects of treatment that are still not agreed upon. There is variation with regards to the "standard" prescription regime of LMWH, with different dose and timing used in the UK, North America and Japan. ${ }^{19}$ This has resulted in conflicting evidence with regards to their safety and efficacy when compared to oral anticoagulants. ${ }^{20}$ Recent meta analyses, conducted with dose adjustments, point towards better safety and efficacy for some of the oral anticoagulant group of medications when compared to LMWH in cancer patients ${ }^{21}$ and lower limb joint arthroplasty patient. ${ }^{22}$ Cost reductions have been suggested for the oral group but the overall savings reflect on home administration regimes ${ }^{23}$ as currently the cost of LMWH per dose is significantly lower that oral medications for the NHS (National Health Service, UK). ${ }^{14}$ The optimal duration of treatment in these groups remains controversial. ${ }^{24}$ The use of anticoagulation medications following surgery has been reported to increase morbidity. ${ }^{25}$

Based on our experience and results, we recommend that patients undergoing surgery using the PRECICE ILLS, should have routine VTE risk assessment on admission. In the absence of contraindications we recommend on the routine use of perioperative contralateral intermittent pneumatic compression stockings, TEDS for 2 weeks following surgery and chemical thromboprophylaxis until the patient is deemed fit for discharge and independently mobile by the physiotherapists. We have found a low incidence of DVT utilising this regime.

\section{Clinical Significance}

The aim of this study was to provide an overview of current practices and highlight the need for developing a consensus of best practice. This will offer further guidance to clinicians and allow for evidence based decision making in order to minimise complications and the potential for litigation as well as optimising cost efficiency of practice. To our knowledge, this is the largest cohort published to date, reporting on the incidence of venous thromboembolism in patients undergoing lower limb intramedullary lengthening.

\section{Acknowledgments}

The authors wish to declare that there has been no external funding for this study. There are no conflicts of interest to declare.

\section{References}

1. Ilizarov GA. The tension-stress effect on the genesis and growth of tissues. Part I. The influence of stability of fixation and soft-tissue preservation. Clin Orthop Relat Res 1989;238:249-281.
2. Ilizarov GA. Transosseous Osteosynthesis - Theoretical and Clinical Aspects and the Regeneration and Growth of Tissue. New York: Springer-Verlag; 1992.

3. Calder PR, Laubscher M, Goodier WD. The role of the intramedullary implant in limb lengthening. Injury 2017;48 (Suppl 1):S52-S58. DOI: 10.1016/j.injury.2017.04.028.

4. Paley D. PRECICE intramedullary limb lengthening system. Expert Rev Med Devices 2015;12(3):231-249. DOI: 10.1586/17434440.2015. 1005604.

5. Flevas DA, Megaloikonomos PD, Dimopoulos L, et al. Thromboembolism prophylaxis in orthopaedics: an update. EFORT Open Rev 2018;3(4):136-148. DOI: 10.1302/2058-5241.3.170018.

6. Lapidus LJ, Ponzer S, Pettersson $\mathrm{H}$, et al. Symptomatic venous thromboembolism and mortality in orthopaedic surgery - an observational study of 45,968 consecutive procedures. BMC Musculoskelet Disord 2013;14:177. DOI: 10.1186/1471-2474-14-177.

7. https://www.nice.org.uk/guidance/ng89/resources/venousthromboembolism-in-over-16s-reducing-the-risk-of-hospitalacquireddeep-vein-thrombosis-or-pulmonary-embolism-pdf-1837703092165.

8. https://www.nice.org.uk/guidance/ng89/resources/department-ofhealth-vte-risk-assessment-tool-pdf-4787149213.

9. Roberts DJ, Panagiotidou A, Sewell M, et al. The incidence of deep vein thrombosis and pulmonary embolism with the elective use of external fixators. Strategies Trauma Limb Reconstr 2015;10(2):67-71. DOI: 10.1007/s11751-015-0219-1.

10. Wagner P, Burghardt RD, Green SA, et al. PRECICE ${ }^{\circ}$ magneticallydriven, telescopic, intramedullary lengthening nail: pre-clinical testing and first 30 patients. SICOT J 2017;3:19. DOI: 10.1051/ sicotj/2016048.

11. Paley D, Debiparshad K, Balci H, et al. Stature Lengthening Using the PRECICE Intramedullary Lengthening Nail. Tech Orthopaed 2015;30(3):167-182. DOI: 10.1097/BTO.0000000000000140.

12. Paley $D$, Harris $M$, Debiparshad K, et al. Limb lengthening by implantable limb lengthening devices. Tech Orthopaed 2014 Jun;29(2):72-85. DOI: 10.1097/BTO.0000000000000072.

13. Calder PR, McKay JE, Timms AJ, et al. Femoral lengthening using the Precice intramedullary limb-lengthening system: outcome comparison following antegrade and retrograde nails. Bone Joint J 2019;101-B(9):1168-1176. DOI: 10.1302/0301-620X.101B9.BJJ-20181271.R1.

14. https://bnf.nice.org.uk/.

15. Anderson FA Jr, Spencer FA. Risk factors for venous thromboembolism. Circulation 2003;107(Suppl 1):I9-I16. DOI: 10.1161/01. CIR.0000078469.07362.E6.

16. Patterson JT, Morshed S. Chemoprophylaxis for venous thromboembolism in operative treatment of fractures of the tibia and distal bones: a systematic review and meta-analysis. J Orthop Trauma 2017;31(9):453-460. DOI: 10.1097/BOT.0000000000000873.

17. Abelseth G, Buckley RE, Pineo GE, et al. Incidence of deep-vein thrombosis in patients with fractures of the lower extremity distal to the hip. J Orthop Trauma 1996;10(4):230-235. DOI: 10.1097/00005131199605000-00002.

18. Wang $\mathrm{H}$, Kandemir $\mathrm{U}$, Liu $\mathrm{P}$, et al. Perioperative incidence and locations of deep vein thrombosis following specific isolated lower extremity fractures. Injury 2018;49(7):1353-1357. DOI: 10.1016/j. injury.2018.05.018.

19. Boyd RA, DiCarlo L, Mandema JW. Direct oral anticoagulants vs. enoxaparin for prevention of venous thromboembolism following orthopedic surgery: a dose-response meta-analysis. Clin Transl Sci 2017;10(4):260-270. DOI: 10.1111/cts.12471.

20. Maratea D, Fadda S, Trippoli $S$, et al. Prevention of venous thromboembolism after major orthopedic surgery: indirect comparison of three new oral anticoagulants. J Thromb Haemost 2011;9(9):1868-1870. DOI: 10.1111/j.1538-7836.2011.04421.x.

21. Dong $Y$, Wang $Y, M a R L$, et al. Efficacy and safety of direct oral anticoagulants versus low-molecular-weight heparin in patients with cancer: a systematic review and meta-analysis. J Thromb Thrombolysis 2019;48(3):400-412. DOI: 10.1007/s11239-019-01871-4. 
22. Kwok CS, Pradhan S, Yeong JK-Y, et al. Relative effects of two different enoxaparin regimens as comparators against newer oral anticoagulants. Meta-analysis and adjusted indirect comparison. Chest 2013;144(2):593-600. DOI: 10.1378/chest.122634.

23. Duran A, Sengupta N, Diamantopoulos A, et al. Cost effectiveness of rivaroxaban versus enoxaparin for prevention of post-surgical venous thromboembolism from a U.S. payer's perspective.
Pharmacoeconomics 2012;30(2):87-101. DOI: 10.2165/11599370000000000-00000.

24. O'Donnell M, Weitz JI.Thromboprophylaxis in surgical patients. Can J Surg 2003;46(2):129-135.

25. Tasker A, Harbord R, BannisterGC. Meta-analysis of low molecularweight heparin versus placebo in patients undergoing total hip replacement and post-operative morbidity and mortality since their introduction. Hip Int 2010;20(1):64-74. DOI: 10.1177/112070001002000110. 